\title{
26375 - DETERMINANT OF COMPLICATIONS WITH FACTOR VIIA THERAPY AFTER CARDIAC SURGERY
}

\section{Wasowicz Marcin PhD, Massimiliano Meineri, MD; Duminda Wijeysundera, MD; Stuart McCluskey, Keyvan Kartkouti, MD; Toronto General Hospital, UHN, Toronto, ONTARIO, Canada}

INTRODUCTION: Blood loss that becomes refractory to standard hemostatic interventions is a serious complication of cardiac surgery that is associated with increased morbidity and mortality1. Recombinant factor VIIa (rFVIIa), a hemostatic agent currently approved for hemophiliac patients, is increasingly being used in the treatment of refractory excessive blood loss (EBL) after cardiac surgery. Its "off label" use in this setting is currently based on several case series and case-control studies that support its effectiveness for this indication. The high rate of serious adverse events in the majority of these reports, however, has raised concerns about its safety in this setting 2 . The purpose of this observational study was to identify the determinants of complications associated with rFVIIa therapy in a cohort of cardiac surgical patients with EBL.

METHODS: In this single-institution observational study, we compared the unadjusted and adjusted perioperative complication rates, expressed as observed to expected $(\mathrm{O} / \mathrm{E})$ ratios, in 114 consecutive cardiac surgical patients who received rFVIIa for refractory EBL with 552 concurrent patients who developed EBL but did not require rFVIIa. The primary outcome was a composite perioperative complication outcome that included death, stroke, renal failure, myocardial infarction, and major vein thrombosis. For risk adjustment, a logistic regression model for this outcome was constructed that adjusted for known confounders.

RESULTS: Whereas the unadjusted complication rate was $88 \%$ higher in patients who received $\mathrm{rFVIIa}(\mathrm{O} / \mathrm{E}=1.88 ; 95 \% \mathrm{CI}=1.58-2.19)$, the adjusted rate was comparable to those who did not receive $\mathrm{rFVIIa}(\mathrm{O} / \mathrm{E}=1.06 ; \mathrm{CI}=0.85-1.28)$. In addition, late versus early rFVIIa therapy (relative to the amount of blood loss with patients dichotomized to greater than or less than the 50th percentile median RBC units transfused before rFVIIa therapy) was associated with an increased risk-adjusted complication rate (late $\mathrm{O} / \mathrm{E}$ $=1.32 ; \mathrm{CI}=1.00-1.63$; early $\mathrm{O} / \mathrm{E}=0.83$; CI $0.54-1.12 ; \mathrm{P}=0.03$ ) (tab.1.).

DISCUSSION: The results of this study suggest that the observed association between rFVIIa therapy and postoperative adverse events is due to the effect of confounders. The results also suggest that rFVIIa therapy late in the course of blood loss may be associated with increased morbidity and mortality. Late rFVIIa therapy may be harmful because as patients bleed more, they are more likely to become hemodynamically unstable and develop disseminated intravascular coagulation (DIC), and rFVIIa therapy in the presence of DIC may increase the risk of thrombotic complications.

REFERENCES: 1. Transfusion 2005;45:26-34. 2. Ann Thorac Surg 2005;80:3-5. 


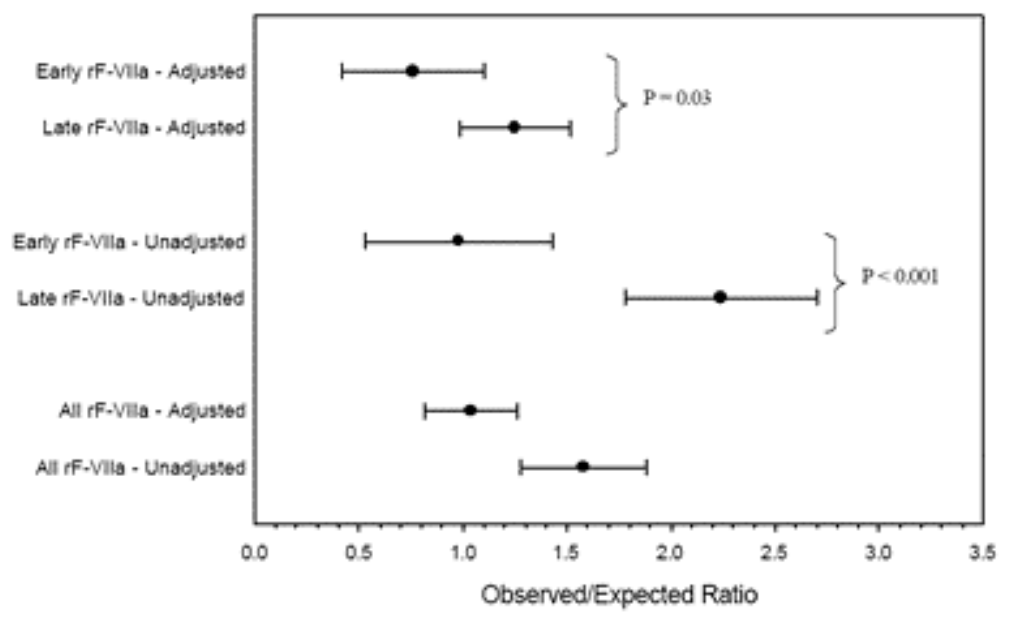

Tab 1. Adjusted and unadjusted perioperative complication rates 